\section{Gliom-Rezidiv: Erfolg der Radiatio vorhersagbar}

\begin{abstract}
Alter, Tumorhistologie und Zeit seit der ersten Bestrahlung geben Auskunft darüber, was eine zweite Bestrahlung gegen ein rezidiviertes Gliom ausrichten kann. Heidelberger Forscher haben aus diesen Parametern einen Prognosescore entwickelt, der die Therapieentscheidung erleichtern kann.
\end{abstract}

nsgesamt 233 Patienten mit rezidivierten Gliomen erhielten eine fraktionierte stereotaktische Radiotherapie (FSRT) mit median 36 Gy in 2 Gy-Einzeldosen. Von den untersuchten Faktoren korrelierten Alter, Tumorhistologie und Zeitraum seit der Erstbestrahlung signifikant mit der Überlebensdauer. In der multivariaten Analyse blieben schließ- lich Alter und Histologie übrig. Keinen Einfluss hatten Tumorvolumen, Karnofsky-Index, neurologische Symptome oder Geschlecht. Der Prognosescore wurde aus den Faktoren Alter, Histologie und Zeit bis zum Rezidiv gebaut; letzterer Parameter aufgrund klinischer Erfahrungen und der in der Literatur verfügbaren Daten. Die Punkte wurden

\begin{tabular}{l|l|}
$\begin{array}{l}\text { Heidelberger Prognosescore } \\
\text { Histologie: }\end{array}$ & Tabelle \\
\hline Alter & $\begin{array}{l}\text { Gliom vom WHO-Grad IV: } 2 \text { Punkte } \\
\text { Gliom vom WHO-Grad III: } 1 \text { Punkt } \\
\text { Gliom vom WHO-Grad II: 0 Punkte }\end{array}$ \\
\hline $\begin{array}{l}\text { O modifiziert nach Combs et al. Acta Oncol. 2012 Jun 11. [Epubahead of print] } \\
\text { < } 1 \text { Punkt }\end{array}$
\end{tabular}

\title{
Überleben mit ZNS-Tumoren in Europa
}

In Europa gibt es nur wenige bevölkerungsbasierte Erhebungen zu Häufigkeit und Überleben von Erwachsenen mit ZNS-Tumoren. Diese EUROCARE-Studie analysierte Inzidenz und 5-Jahres-Überlebensraten maligner und benigner ZNS-Tumoren und deren Veränderungen in einem 14-Jahreszeitraum.

$\mathrm{D}$ ie jüngsten auswertbaren Daten stammten aus den Jahren 2002/2003. Für Überlebenszeittrends konnten die Zahlen von 24 Krebsregistern mit kontinuierlicher Erfassung seit 1988 herangezogen werden. Die ZNS-Tumoren wurden nach morphologischen Kriterien in 14 verschiedene Tumorkategorien unterteilt. Darüber hinaus wurden Geschlecht, Alter bei Diagnose und Region (Großbritannien [UK], Irland, Nord-, Zentral-, Ost- und Südeuropa) erfasst. Die deutschen Daten entstammen dem saarländischen Krebsregister.

Das relative 5-Jahres-Gesamtüberleben betrug für benigne Tumoren $85 \%$, für maligne $19,9 \%$. Bei den benignen Tumoren rangierte es zwischen 90,6\% (Nordeuropa) und 77,4\% (UK, Irland), bei malignen Tumoren zwischen $25,1 \%$ (Nordeuropa) und 15,6\% (UK, Irland).

Das Überleben nahm mit dem Alter bei Diagnose ab und war besonders günstig in der Gruppe der 15- bis 29-Jährigen.

Bei den glialen Tumoren zeigte sich eine große Bandbreite im 5-JahresÜberleben je nach Morphologie: 83,5\% für Ependymome und Tumoren des Plexus chorioideus vs. 2,7\% für Glioblastome. Für Neurinome lag es bei 96,5\%, für primitive neuroektodermale Tumoren und Medulloblastome bei $44,9 \%$.

Die Überlebensunterschiede zwischen den verschiedenen Regionen verringerten sich deutlich nach Berücksichtigung der Morphologie. Allerdings blieb bei Patienten aus Großbritannien, Irland und Osteuropa ein 30-40\% höheres Sterblichkeitsrisiko im Vergleich zu Nordeuropa bestehen. Auch in Zentraleuropa blieb das Sterblichkeitsrisiko gegenüber Nordeuropa um $20 \%$ erhöht. wie in der Tabelle gezeigt vergeben, und das führt zu folgender Bewertung: 0 Punkte: exzellente Prognose (Überleben nach 6, 12, 24 und 36 Monaten in Prozent: 89, 73, 50, 35)

1 Punkt: gute Prognose $(82,74,41,23)$ 2 Punkte: mäßige Prognose $(68,50,25,11)$ 3 Punkte: schlechte Prognose $(68,20,6,3)$ 4 Punkte: schlechte Prognose $(72,28,8,4)$

Fazit: Aus den Parametern Alter, Histologie des Glioms und Zeit seit der Erstbestrahlung lässt sich ein Wert errechnen, der Auskunft über die Chancen einer zweiten Radiatio im Rezidiv gibt. Den Autoren zufolge sollte er aber nicht allein den Ausschlag geben: „Man muss immer bedenken, dass über die Therapie individuell zu entscheiden ist.“" Es könnten durchaus auch Faktoren einfließen, die das Therapieergebnis nicht unmittelbar beeinflussten.

Robert Bublak

Combs SE et al. Generation and validation of a prognostic score to predict outcome after reirradiation of recurrent glioma. Acta Oncol. 2012 Jun 11. [Epub ahead of print].

Das Überleben von Patienten mit benignen ZNS-Tumoren stieg im untersuchten 14-Jahres-Zeitraum von 69,3\% (1988-1990) auf 77,1\% (2000-2002), wahrscheinlich durch frühere und verfeinerte Diagnostik. Hingegen verbesserte es sich bei malignen ZNS-Tumoren nicht - trotz deutlicher Veränderungen bei anderen soliden Tumoren in dieser Zeit. Allerdings wurden neue Therapieverfahren und Medikamente (z.B. Temozolomid) erst nach 2002 eingeführt.

Fazit: Unterschiede im 5-Jahres-Überleben zwischen Europas Regionen gehen weitgehend auf Variationen in der Häufigkeitsverteilung von ZNS-Tumoren zurück. Andererseits lässt sich ein Einfluss sozioökonomischer Faktoren (Osteuropa) oder eines verzögerten Zugangs zur Diagnostik (UK) nicht von der Hand weisen. Über 14 Jahre verbesserte sich das Überleben bei malignen Tumoren nicht.

Brigitte Schalhorn

Sant M et al. Survival of European patients with central nervous system tumors. Int J Cancer. 2012;131(1):173-85. 